Z Gerontol Geriat 2016 · 49:349

DOI 10.1007/s00391-016-1074-5

Online publiziert: 2. Juni 2016

๑) Springer-Verlag Berlin Heidelberg 2016

CrossMark

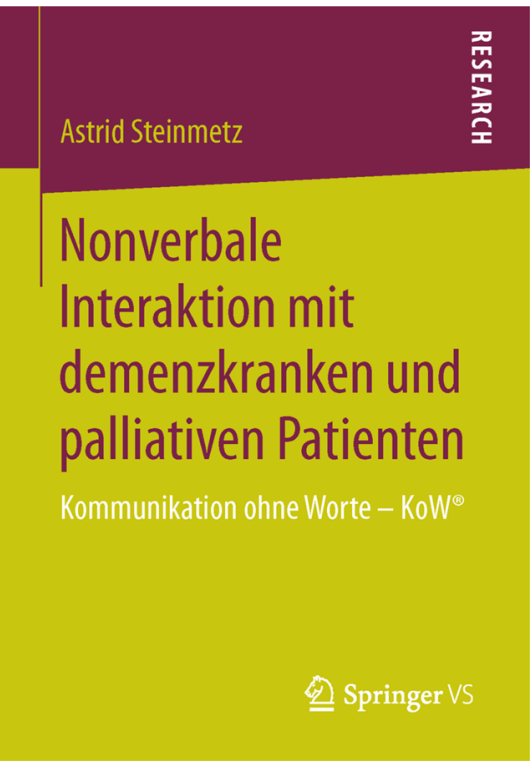

\section{Originalpublikation}

Steinmetz A (2016) Nonverbale Interaktion mit demenzkranken und palliativen

Patienten. Kommunikation ohne Worte $\mathrm{KoW}^{\circledR}, 1$. Aufl. Springer VS, Wiesbaden. XXII, 263 S., 2 Abb., Softcover, EUR 39,99 (D), ISBN13: $978-3658113339$

Es handelt sich um die Veröffentlichung einer Dissertation im Fach Gerontologie an der Fakultät für Verhaltens- und Kulturwissenschaften der Ruprecht-KarlsUniversität Heidelberg aus dem Jahr 2014. Darin geht es um die Fundierung und Evaluierung eines Trainingsprogramms zur Förderung von dialogischer Interaktion mit demenzkranken und palliativen Patienten. Die Autorin tritt mit einer gründlichen empirischen Forschungsmethodik den - auf den ersten Blick - ungewöhnlich erscheinenden Beweis an, dass bei krankheitsbedingten kommunikativen Einschränkungen eine dialogische Begegnung möglich ist. Neu-

\author{
A. Zieger \\ Oldenburg, Deutschland
}

\title{
Dialogisches Prinzip in der Patienteninteraktion
}

rologisch schwerstkranke sowie von der verbalen Kommunikation und Interaktion abgeschnittene Menschen mit Demenz sind existenziell auf Dialogpartner angewiesen. Dies betrifft nicht nur Patienten auf Palliativ- und Hospizstationen, nicht nur am Ende des Lebens, sondern auch Patienten nach einem Unfall oder einem Herz-Kreislauf-Stillstand mit Sauerstoffmangel des Gehirns im Koma und im Wachkoma. Es gilt, ihre besonderen, verborgenen und oft nur körpersprachlich erkennbaren Möglichkeiten des Verstehens und Antwortens zu erkennen, wahrzunehmen und zu nutzen. Im beziehungsmedizinischen, pflegerischen und therapeutischen Kontext, sei es auf der Intensivstation, in der Frührehabilitation, auf Wachkomastationen, im Altenpflegeheim oder in Palliativ- und Hospizeinrichtungen, sind in den letzten 20 Jahren diverse nonverbale Kommunikations- und Interaktionszugänge, die diese Wahrnehmung üben, sensibilisierend erschlossen worden [1]. Beispiele sind die basale Stimulation in der Pflege [2] und die Erforschung des körpernahen Dialogaufbaus in der neurologischen Intensiv- und Frührehabilitationsmedizin [3]. Dies geschah ebenfalls in Form einer sich im Bereich der Sonder- und Rehabilitationspädagogik mit körpernahen und -fernen Techniken etablierenden unterstützten Kommunikation (UK) mitsamt eines ganzen Lehr- und Forschungsfelds [4]. Doch gab es für den nonverbalen Dialog bisher nicht den empirischen Nachweis der Wirksamkeit, die über persönliche Erfahrung, Intuition und therapeutisches Übertragungsgeschehen hinausgeht, und auch insbesondere der Erlernbarkeit solcher Fähigkeiten. Dies ist nun der Autorin des vorgestellten Buchs unter Anwendung empirischer
Untersuchungsmethoden und der Evaluierung des von ihr selbst entwickelten Trainingsprogramms „Kommunikation ohne Worte KoW ${ }^{\circledR “ " ~ g e l u n g e n . ~ D a s ~ F a c h-~}$ buch schließt somit eine empfindliche Erkenntnislücke, die über den Umgang mit den demenzkranken und den palliativ versorgten Patienten als demografisch häufige und relevante Problemgruppen mit schwer eingeschränktem Kommunikationsvermögen hinausgeht. Der nonverbale Dialog ist auch für die Versorgung und Teilhabe der oben genannten anderen, selteneren, aber nicht minder existenziell schwer betroffenen neurologischen Problemgruppen und ihre Angehörigen erlernbar!

\section{Korrespondenzadresse}

\section{Prof. Dr. A. Zieger}

Ziegelhofstr. 57, 26121 Oldenburg, Deutschland

a.zieger@t-online.de

Interessenkonflikt. A. Zieger gibt an, dass kein Interessenkonflikt besteht.

\section{Literatur}

1. Otterstedt C (2005) Der nonverbale Dialog. Für Begleiter von Schwerkranken, Schlaganfall, Komapatienten und DemenzBetroffenen mit Übungen zur Wahrnehmungssensibilisierung. modernes Lernen, Dortmund

2. Bienstein C, Fröhlich A (2003) Basale Stimulation in der Pflege. Die Grundlagen. Kallmeyer, Seelze Velber

3. Zieger A (1996) Dialogaufbau in der Frühphase mit komatösen Schädel-Hirn-Verletzten. In: Lipp B, Schlaegel W, Kammerer T, Ingensiep HW, Rehbock T (Hrsg) "Wege von Anfang an“. Frührehabilitation schwerst hirngeschädigter Patienten. Neckar, Villingen Schwenningen, 237-246 (in Grenzsituationen)

4. Bollmeyer $\mathrm{H}$ et al (Hrsg) (2011) UK inklusive. Teilhabe durch Unterstützte Kommunikation. von Loeper, Karlsruhe 